\title{
Verification of Differential Low Noise Amplifier Measurement
}

\author{
M. Panahi and D. George
}

\begin{abstract}
MACS Research Group, School of Electrical and Electronic Engineering, The University of Manchester, P.O. Box 88, Sackville Street Building, Sackville Street, Manchester, UK, M60 1QD. *
\end{abstract}

\begin{abstract}
Differential input to single ended output Low Noise Amplifiers (LNA) designs and fabrications are required for the front end of 2-PAD project in SKADS. The differential input for these LNAs are essential to match the differential output of the Bunny Ear Comb-line Antenna (BECA) connected before these LNAs.

A low noise amplifier has been designed, fabricated and tested for 2-PAD project. Detailed explanation of this designed amplifier and its results will be described.

A commercially available amplifier was used to devise a robust test plan to measure s-parameter responses and noise of various topologies of LNA designs for 2-PAD LNAs.

This commercially available amplifier is ideal for this study as it allows us to use it in three different modes of operation including single ended input to differential output, differential input to differential output and differential input to single ended output. Therefore the later mode of operation is our interest.

S-parameter response tests were carried out for the differential input to differential output and differential input to single ended output modes of this amplifier using a 4 port vector network analyzer.

A noise measurement model for differential amplifiers was proposed by the author in more detail in Bhaumik et al. (2009). The commercially available amplifier and the designed LNAs operate in the same frequency range as well as having differential input and single ended output feature; therefore these tests and method of measurements are particularly useful for the reason that can be utilized in measuring the low noise amplifiers designed for 2-PAD project.

As a result, the procedure used for measuring the differential input to single ended output operation mode of the commercially available amplifier would verify the precision and suitability of this method for the designed LNAs.
\end{abstract}

\section{Introduction}

One of the various undergoing verification programmes in SKADS is 2-PAD (Dual-Polarization All digital Phased Array) which covers the frequency range of $0.3-1 \mathrm{GHz}$. [Schillizi et al. (2007)]

A commercially available amplifier with four ports, two input ports and two output ports was used for the S-parameter responses measurement purpose. This amplifier is illustrated in Fig. 1. This amplifier can be figured to operate in three different modes: 1) differential input to differential output, 2) differential input to single ended output by using a transformer \& 3) single ended input to differential output. Modes 1 and 2 were concentrated on because of their differential input feature which is a requirement for our designed LNAs in the front end section to match the differential output impedance of the Bunny Ear Comb line Antenna (BECA).

\section{Mixed Mode Scattering Parameters}

The concept of mixed mode $\mathrm{S}$-parameters are driven from basic S-Parameter definition (Bockelman \& Eisenstadt 1995). Mixed mode scattering parameters explain the differential and common mode responses to differential and common mode drives and are useful to be in balanced devices (Martens et al. 2005).

* This work was supported by the European Commission Framework Program 6, Project SKADS, Square Kilometre Array Design Studies (SKADS), contract no 011938.

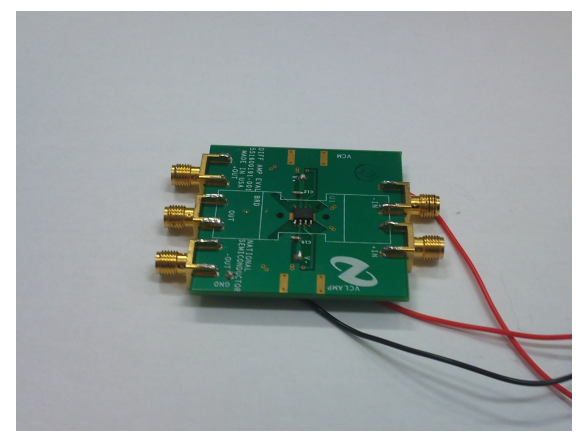

Fig. 1: Commercially available amplifier.

The mixed mode S-parameter of a four port device is given in matrix below (Agilent 2007). Thus this matrix is valid for an amplifier with differential input to differential output.

$$
\left[\begin{array}{llll}
S_{d d 11} & S_{d d 12} & S_{d c 11} & S_{d c 12} \\
S_{d d 21} & S_{d d 22} & S_{d c 21} & S_{d c 22} \\
S_{c d 11} & S_{c d 12} & S_{c c 11} & S_{c c 12} \\
S_{c d 21} & S_{c d 22} & S_{c c 21} & S_{c c 22}
\end{array}\right]
$$

The mixed mode $\mathrm{S}$-parameter of a three port device is given in matrix below (Agilent 2007). Therefore this matrix would be valid for an amplifier with differential input and single-ended output. 


$$
\left[\begin{array}{lll}
S_{s s 11} & S_{s d 12} & S_{s c 12} \\
S_{d s 21} & S_{d d 22} & S_{d c 22} \\
S_{c s 21} & S_{c d 22} & S_{c c 22}
\end{array}\right]
$$

Since the main focus was on modes 1 and 2 of the amplifier (See Section 1), their measured mixed mode s-parameter result is explained in more detail in Sections 3 and 4 respectively.

\section{Testing the Commercially Available Amplifier with Differential Input and Differential Output}

The commercially available amplifier was constructed to perform with differential input and differential output to enable us to study the RF performance of a fully differential amplifier. The S-parameter responses of this amplifier were measured by a four port VNA over the frequency range of $10-1000 \mathrm{MHz}$.

Measured gain of this amplifier with differential input and differential output $\left(S_{d d 21}\right)$, is illustrated in Fig. 2.

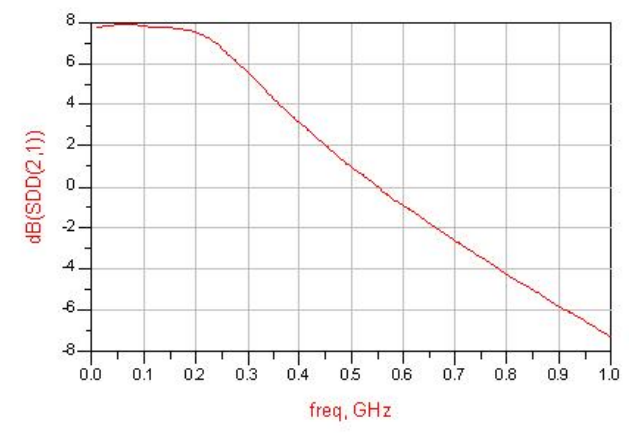

Fig. 2: Measured S-parameter response that corresponds to the gain of the commercially available amplifier in fully differential mode.

The maximum gain $\left(S_{d d 21}\right)$ derived from this amplifier in the fully differential mode is $8 \mathrm{~dB}$ up to $0.2 \mathrm{GHz}$.

\section{Testing the Commercially Available Amplifier with Differential Input and Single-Ended output}

Characterizing the differential input and single ended Low Noise Amplifiers (LNA) is a challenging process for 2-PAD project. Therefore, a commercially available LNA (Fig. 1) was used to verify the measurement procedure of a differential input to single ended output amplifier. Mixed mode s-parameter responses characterization of this amplifier was measured by a four port Vector Network Analyzer (VNA). Measured mixed mode s-parameter $S_{s d 12}$ of the commercially available amplifier is shown in Fig. 3 over the frequency range of $10 \mathrm{MHz}$ to 1000MHz. As shown in Fig. 3, the maximum gain measured with this configuration was more that $10 \mathrm{~dB}$ up to $0.2 \mathrm{GHz}$.

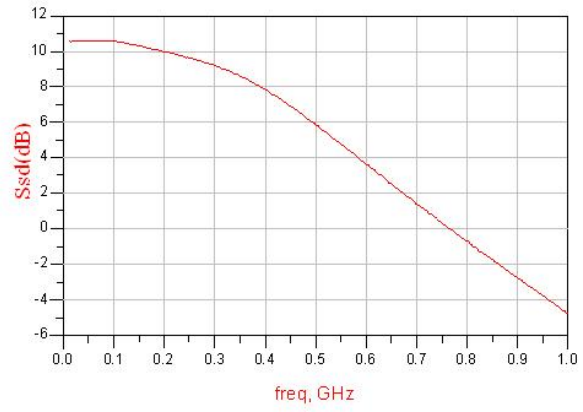

Fig. 3: Measured mixed mode S-parameter response of $S_{s d 12}$ for a three port amplifier.

\section{Differential Input and Single-Ended Amplifier Design}

A Low Noise Amplifier with two stages was designed in Advanced Design System (ADS) as part of 2-PAD project.(Agilent 2009)

Fig. 4 shows a block diagram of a differential input and single ended low noise amplifier which was designed for the front end of the 2-PAD project.

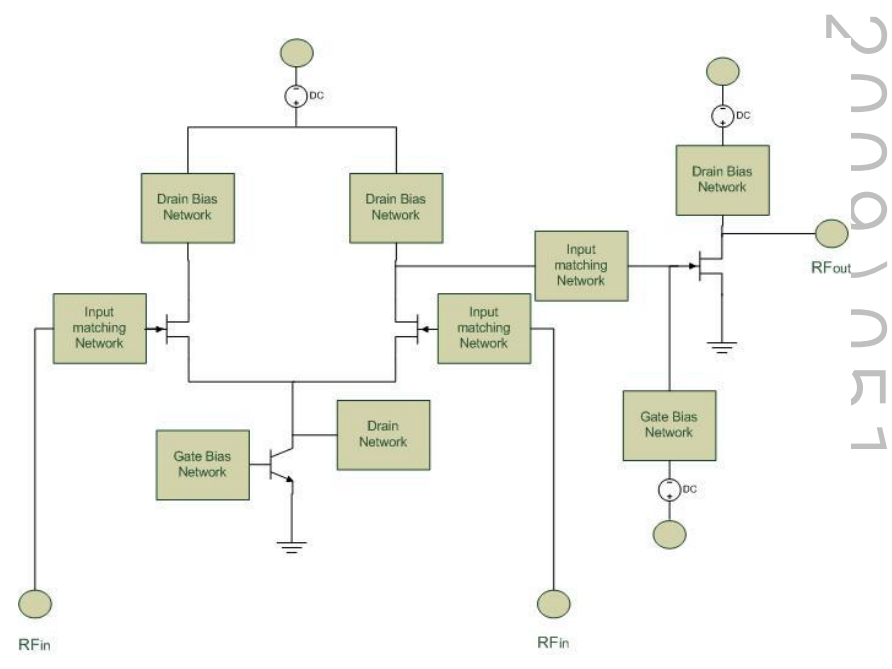

Fig. 4: Block diagram of designed differential LNA

This LNA operates in the frequency range of 0.5 to $1 \mathrm{GHz}$. This LNA has a 150 ohm differential input to match the BECA antenna impedance before the LNA and a $50 \mathrm{ohm}$ single ended output. Simulated S-parameters of the designed differential LNA is illustrated in Fig. 5.

According to the simulated S-parameter responses for this amplifier (Fig. 5), the input return loss is better than $10 \mathrm{~dB}$ between 0.5 to $0.8 \mathrm{GHz}$. Gain produced by this amplifier varies between $32.5 \mathrm{~dB}$ to $26 \mathrm{~dB}$ over the frequency range of 0.5 $1 \mathrm{GHz}$. The simulated noise figure of this amplifier is less than $1 \mathrm{~dB}$ up to $0.9 \mathrm{GHz}$. 

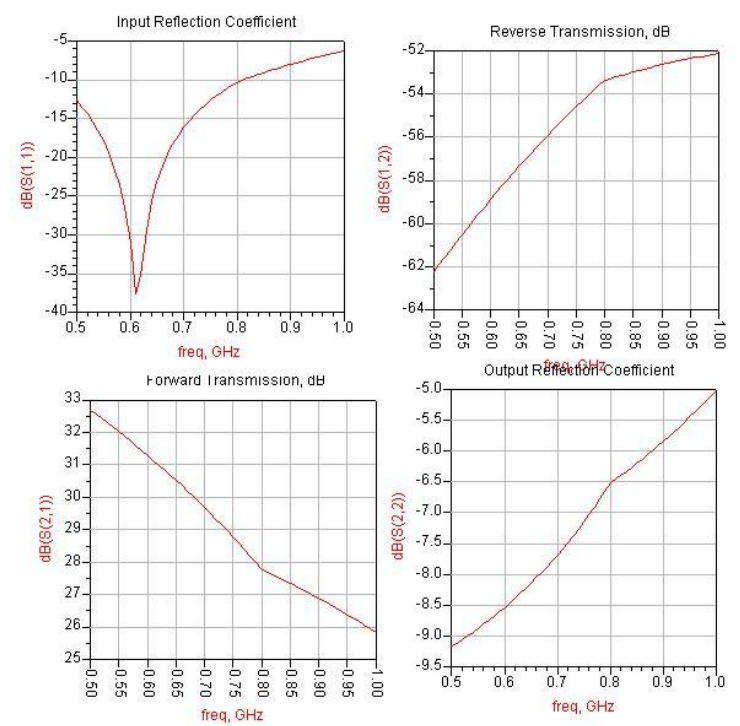

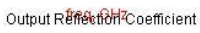

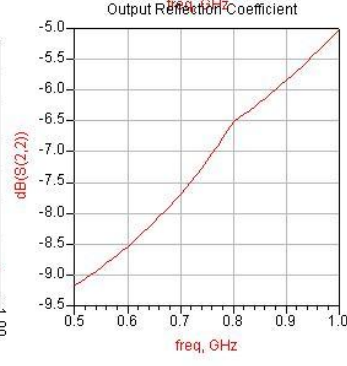

Fig. 5: Simulated S-parameter responses of the differential amplifier.

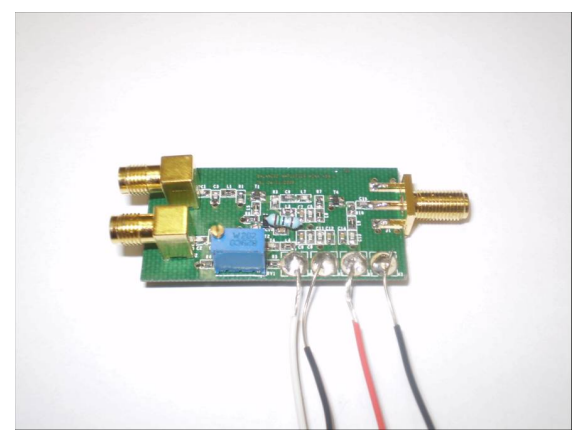

Fig. 6: Manufactured differential input and single ended output LNA.

Designed differential input to single ended output amplifier was fabricated as shown in Fig. 6. It consists of ATF33143 commercial pHEMTs transistors with NF of $0.5 \mathrm{~dB}$ and gain of $15 \mathrm{~dB}$ from Avago Technologies. 3 volts DC voltage was supplied for this amplifier. The mixed mode s-parameter measurement method was applied on this LNA using the same four port VNA but no gain was delivered from designed LNA due to an oscillation problem within the LNA itself. These problems are still under investigation.

\section{Conclusions}

An amplifier design with differential input and single ended output has been described for the front end of the 2-PAD programme. This amplifier operates over the frequency range of $0.5-1 \mathrm{GHz}$. Simulated RF performance, fabricated prototype and testing results are explained above. A commercially available LNA which can be derived as either fully differential LNA or differential input to single-ended output LNA was under in- vestigation to verify a method for measuring the scattering parameters of the designed LNA in 2-PAD programme.

Acknowledgements. We would like to thank Dr. Georgina Harris and Tim Ikin in Jodrell Bank Observatory for their help and support during fabrication of designed LNAs.

\section{References}

Agilent. 2007, E5070B\&E5071B Series RF Network Analyzer User's Guide, 11th Ed.

Agilent. 2009, Advanced Design System(ADS)

Bhaumik, S., Panahi, M., \& Kettle, D. 2009, in Antennà Technology and Applied Electromagnetics and the Canadian Radio Science Meeting, 2009. ANTEM/URSI 2009. 13th International Symposium on, 1-4

Bockelman, D. \& Eisenstadt, W. 1995, Microwave Theory and Techniques, IEEE Transactions on, 43, 1530

Martens, J., Judge, D., \& Bigelow, J. 2005, Microwave Magazine, IEEE, 6, 72

Schillizi, R. T., Alexander, P., Cordes, J. M., et al. 2007, Memo 100: Preliminary Specifications for the Square Kilometre Array., Accessed on 24 Nov2009 